\title{
INVARIANCE OF THE CAUCHY MEAN-VALUE EXPRESSION WITH AN APPLICATION TO THE PROBLEM OF REPRESENTATION OF CAUCHY MEANS
}

\author{
LUCIO R. BERRONE
}

Received 30 August 2004 and in revised form 11 May 2005

The notion of invariance under transformations (changes of coordinates) of the Cauchy mean-value expression is introduced and then used in furnishing a suitable two-variable version of a result by L. Losonczi on equality of many-variable Cauchy means. An assessment of the methods used by Losonczi and Matkowski is made and an alternative way is proposed to solve the problem of representation of two-variable Cauchy means.

\section{Introduction and preliminaries}

Let $f, g$ be two differentiable real functions defined on an open interval $I$. Let us write the expression corresponding to the classical Cauchy mean-value theorem in the form

$$
\frac{f(y)-f(x)}{g(y)-g(x)}=\frac{f^{\prime}(\mu(x, y))}{g^{\prime}(\mu(x, y))}, \quad x, y \in I .
$$

A sense is conveyed to this particular way of writing by assuming that the quotient $f^{\prime} / g^{\prime}$ is a strictly monotone function, so that the Cauchy "intermediate value" $\mu(x, y)$ turns out to be a uniquely determined function of the pair of variables $x, y \in I$. In this situation, a (differentiable) transformation $\Phi: \mathcal{U} \rightarrow \mathbb{R}^{2}$ defined on a plane region $\boldsymbol{U} f(I) \times g(I)$ and specified by $\Phi(x, y)=(X(x, y), Y(x, y))$ is said to leave expression (1.1) invariant for the curve $(f(t), g(t))$ when (1.1) is satisfied by the plane curve $\Phi(f(t), g(t))=(F(t), G(t))$ with the same intermediate value $\mu$. In other words, the transformation $\Phi=(X, Y)$ leaves expression (1.1) invariant for the curve $(f(t), g(t))$ provided that

$$
\frac{X(f(y), g(y))-X(f(x), g(x))}{Y(f(y), g(y))-Y(f(x), g(x))}=\frac{X_{x}(f(\mu), g(\mu)) f^{\prime}(\mu)+X_{y}(f(\mu), g(\mu)) g^{\prime}(\mu)}{Y_{x}(f(\mu), g(\mu)) f^{\prime}(\mu)+Y_{y}(f(\mu), g(\mu)) g^{\prime}(\mu)},
$$

when $\mu=\mu(x, y)$ is specified by

$$
\frac{f(y)-f(x)}{g(y)-g(x)}=\frac{f^{\prime}(\mu)}{g^{\prime}(\mu)}
$$

May be the reader prefer instead to say that it is the intermediate value $\mu$ of the expression (1.1) that is invariant under the transformation $\Phi$. In any case, our choice of 
the terminology is motivated by the fact that (1.1) remains unchanged when $(f, g)$ is replaced by $(F, G)=\Phi(f, g)$.

Now, a given transformation $\Phi: \mathbb{R}^{2} \rightarrow \mathbb{R}^{2}$ can leave expression (1.1) invariant for every curve $(f(t), g(t))$. In this case, we will plainly say that $\Phi$ leaves expression (1.1) invariant. For instance, it is easy to see that all planar affine transformations

$$
\begin{aligned}
& X(x, y)=a_{1} x+b_{1} y+c_{1}, \\
& Y(x, y)=a_{2} x+b_{2} y+c_{2},
\end{aligned}
$$

where $a_{i}, b_{i}, c_{i} \in \mathbb{R}(i=1,2) a_{1} b_{2}-a_{2} b_{1} \neq 0$, leave expression (1.1) invariant. In fact, assuming that (1.3) holds, for $x, y \in I$, we have

$$
\begin{aligned}
\frac{X(f(y), g(y))-X(f(x), g(x))}{Y(f(y), g(y))-Y(f(x), g(x))} & =\frac{\left(a_{1} f(y)+b_{1} g(y)+c_{1}\right)-\left(a_{1} f(x)+b_{1} g(x)+c_{1}\right)}{\left(a_{2} f(y)+b_{2} g(y)+c_{2}\right)-\left(a_{2} f(x)+b_{2} g(x)+c_{2}\right)} \\
& =\frac{a_{1}(f(y)-f(x))+b_{1}(g(y)-g(x))}{a_{2}(f(y)-f(x))+b_{2}(g(y)-g(x))} \\
& =\frac{a_{1}((f(y)-f(x)) /(g(y)-g(x)))+b_{1}}{a_{2}((f(y)-f(x)) /(g(y)-g(x)))+b_{2}} \\
& =\frac{a_{1}\left(\left(f^{\prime}(\mu(x, y))\right) /\left(g^{\prime}(\mu(x, y))\right)\right)+b_{1}}{a_{2}\left(\left(f^{\prime}(\mu(x, y))\right) /\left(g^{\prime}(\mu(x, y))\right)\right)+b_{2}} \\
& =\frac{X_{x}(f(\mu), g(\mu)) f^{\prime}(\mu)+X_{y}(f(\mu), g(\mu)) g^{\prime}(\mu)}{Y_{x}(f(\mu), g(\mu)) f^{\prime}(\mu)+Y_{y}(f(\mu), g(\mu)) g^{\prime}(\mu)} .
\end{aligned}
$$

It must be emphasized that the notion of invariance of expression (1.1) for a curve $(f(t), g(t))$ is not a geometric one, in the sense that it depends on the parameterization of the curve. Unlike what happens with invariance of expression (1.1) for a given curve $(f(t), g(t))$, plain invariance of expression (1.1) is a geometric concept (does not depend on parameterizations). We will return to the question of parameterization at the end of the paper. On the other hand, if a transformation $\Phi_{1}$ leaves expression (1.1) invariant for a given curve $(f(t), g(t))$ and a second transformation $\Phi_{2}$ leaves expression (1.1) invariant for the curve $\Phi_{1}(f(t), g(t))$, then the composite transformation $\Phi_{2} \circ \Phi_{1}$ also leaves expression (1.1) invariant for the curve $(f(t), g(t))$. Furthermore, if $\Phi$ leaves expression (1.1) invariant for the curve $(f(t), g(t))$, it is not difficult to see that $\Phi^{-1}$ leaves expression (1.1) invariant for $\Phi(f(t), g(t))$. Thus, a simple argument shows that the set of transformations that leave expression (1.1) invariant constitutes a group under composition. Our subsequent developments will be directed to determine this group. Indeed, the bulk of this paper will consist of a minute proof of the following result.

THEOREM 1.1. The affine group (1.4) is the most general group of $\mathscr{C}^{2}$ transformations that leave expression (1.1) invariant.

A proof for this theorem is the subject matter of the following three sections (Sections 2, 3, and 4), while in Section 5, an application is found discussing the recent Losonczi's 
results on representation of Cauchy means. In this way, a few paragraphs devoted to this general class of means and to the related problem of their representation are in order.

Given a pair $f, g$ of continuous and strictly monotonic functions defined on an interval $I \subseteq \mathbb{R}$, its associated Cauchy mean $\left[{ }_{g}^{f}\right]$ is the continuous symmetric mean defined on $I \times I$ by $(\mathrm{cf} .[4])$

$$
\left[\begin{array}{l}
f \\
g
\end{array}\right](x, y) \triangleq \begin{cases}f^{-1}\left(\frac{1}{g(y)-g(x)} \int_{x}^{y} f(\xi) d g(\xi)\right) & \text { if } x \neq y \\
x & \text { otherwise }\end{cases}
$$

where the integral appearing in the right-hand side is a Riemann-Stieltjes integral. For the general notion of continuous symmetric mean, we refer to the treatises $[1,7]$ (see also $[3,4])$. Assuming that the function $g$ in (1.6) is differentiable and setting, for a fixed $a \in I$,

$$
\begin{gathered}
F(t) \triangleq \int_{a}^{t} f(\xi) d g(\xi)=\int_{a}^{t} f(\xi) g^{\prime}(\xi) d \xi, \quad y \in I, \\
G(t) \triangleq g(t), \quad t \in I,
\end{gathered}
$$

the expression

$$
\frac{F(y)-F(x)}{G(y)-G(x)}=\frac{F^{\prime}(\mu(x, y))}{G^{\prime}(\mu(x, y))}
$$

of the Cauchy mean-value theorem is recovered from (1.6) with $\mu(x, y)=\left[{ }_{g}^{f}\right](x, y)$ : the definition of the mean $\left[{ }_{g}^{f}\right]$ is then related to the Cauchy mean-value theorem and the sense of the used terminology becomes evident.

Many important classes of means arise as subclasses of Cauchy means by suppressing, so to speak, "one degree of (functional) freedom" in definition (1.6). Thus, for example, the subclass of Cauchy means of the form $\left[\begin{array}{l}f \\ f\end{array}\right]$ coincides with the class of quasiarithmetic means (see [7, Chapter 4]). In fact, for $x, y \in I, x \neq y$, we have

$$
\left[\begin{array}{l}
f \\
f
\end{array}\right](x, y)=f^{-1}\left(\frac{1}{f(y)-f(x)} \int_{x}^{y} f(\xi) d f(\xi)\right)=f^{-1}\left(\frac{f(x)+f(y)}{2}\right) .
$$

The subclasses of Cauchy means of the forms $\left[\begin{array}{c}f \\ i d_{I}\end{array}\right]$ and $\left[\begin{array}{c}i d_{I} \\ f\end{array}\right]$, where $i d_{I}$ denotes the identity function defined on the interval $I$, are particularly simple. The first one of them, the class of Lagrangian means (see $[3,7,13]$ ), is to the Lagrange mean-value theorem as the entire class of Cauchy means is to Cauchy's theorem. In its turn, the means of the form $\left[\begin{array}{c}i d_{I} \\ f\end{array}\right]$ were named anti-Lagrangian means and were studied in [4].

A generic member of any class of the just-mentioned subclasses of means turns out to be clearly identified once a single continuous and strictly monotonic function $f$ is specified on the interval $I$, and accordingly it will be denoted by $[f]$ in the sequel. Using this notation, the problem of representation asks for conditions of validity of an equality like

$$
\left[f_{1}\right]=\left[f_{2}\right]
$$


in a given subclass of means. In other words, given two means $\left[f_{1}\right],\left[f_{2}\right]$ belonging to a fixed class, say the class of Lagrangian means, necessary and sufficient conditions must be found (on the functions $f_{1}, f_{2}$ ) in order that the equality (1.10) should hold. The problem of representation is satisfactorily solved for all of the three above-mentioned subclasses of means. Furthermore, the solution is the same in every case: two quasiarithmetic [Lagrangian, anti-Lagrangian] means satisfy equality (1.10) if and only if the functions $f_{1}, f_{2}$ are related to each other through

$$
f_{2}=\alpha f_{1}+\beta
$$

with $\alpha, \beta \in \mathbb{R}, \alpha \neq 0$. Different approaches were used to prove these facts; for details, we refer the reader to $[3,4,7,13]$ and to the references cited in these sources.

The fact expressed by (1.11) may be rephrased by saying that $\left[f_{1}\right]=\left[f_{2}\right]$ if and only if $f_{2}$ is the image of $f_{1}$ under a real affine transformation. Now, taking into account the behavior of Cauchy means under conjugacy, which is established by the formula

$$
\phi^{-1} \circ\left[\begin{array}{l}
f \\
g
\end{array}\right] \circ(\phi \times \phi)=\left[\begin{array}{l}
f \circ \phi \\
g \circ \phi
\end{array}\right]
$$

where $\phi: I \rightarrow I$ is a homeomorphism, we realize that the problem of representation of "one degree of (functional) freedom" Cauchy means can be similarly solved. As a matter of fact, the equality

$$
\left[\begin{array}{l}
f_{1} \\
g
\end{array}\right]=\left[\begin{array}{l}
f_{2} \\
g
\end{array}\right]
$$

holds if and only if

$$
\left[\begin{array}{c}
f_{1} \circ g \\
i d_{I}
\end{array}\right]=g^{-1} \circ\left[\begin{array}{l}
f_{1} \\
g
\end{array}\right] \circ(g \times g)=g^{-1} \circ\left[\begin{array}{l}
f_{2} \\
g
\end{array}\right] \circ(g \times g)=\left[\begin{array}{c}
f_{2} \circ g \\
i d_{I}
\end{array}\right]
$$

or, in view of (1.11), if and only if there exist two real constants $\alpha \neq 0$ and $\beta$ such that

$$
f_{2} \circ g=\alpha\left(f_{1} \circ g\right)+\beta
$$

Analogously, we see that (1.15) also provides a necessary and sufficient condition in order that

$$
\left[\begin{array}{l}
g \\
f_{1}
\end{array}\right]=\left[\begin{array}{l}
g \\
f_{2}
\end{array}\right]
$$

Summarizing the above discussion, we can say that, after identity (1.12), conjugacy amounts the same as reparameterization, and therefore we cannot at all be surprised by a consequence like (1.15) of this fact. 
As recognized by Losonczi in [11], the full problem of representation of two-variable Cauchy means presents a considerable difficulty. Indeed, necessary and sufficient conditions on the pairs $f, g$ and $F, G$ under which the equality

$$
\left[\begin{array}{l}
f \\
g
\end{array}\right]=\left[\begin{array}{l}
F \\
G
\end{array}\right]
$$

should hold have been obtained in [11] for many- (more than two) variable Cauchy means. Such a generalization of two-variable Cauchy means is obtained from suitable generalizations of the Cauchy mean-value theorem like that one by Leach and Sholander (see [9]):

$$
\frac{\left[x_{1}, x_{2}, \ldots, x_{n}\right]_{f}}{\left[x_{1}, x_{2}, \ldots, x_{n}\right]_{g}}=\frac{f^{(n-1)}\left(\mu\left(x_{1}, x_{2}, \ldots, x_{n}\right)\right)}{g^{(n-1)}\left(\mu\left(x_{1}, x_{2}, \ldots, x_{n}\right)\right)},
$$

where $x_{1}, x_{2}, \ldots, x_{n} \in I$ and, for a given function $h$ possessing at least $(n-1)$-order derivatives on $I$, the symbol $\left[x_{1}, x_{2}, \ldots, x_{n}\right]_{h}$ denotes the divided difference of $h$ at the points $x_{1}, x_{2}, \ldots, x_{n}$ (see $[8$, pages 18-20]). As openly suggested by the way of writing of (1.18), the unique (functional) determination of the (many-variable) Cauchy mean $\mu$ in (1.18) depends on the inversibility of the quotient $f^{(n-1)} / g^{(n-1)}$.

Let us denote by $\left[{ }_{g}^{f}\right]\left(x_{1}, x_{2}, \ldots, x_{n}\right)$ the $n$-variables Cauchy mean $\mu$ defined by (1.18). The main result in [11] reads as follows.

Theorem 1.2. Let $n \geq 3$ and let $f, g, F, G$ be four real functions defined on $I$ such that

(i) $f, g, F, G$ are $(n+2)$ times continuously differentiable on $I$,

(ii) $g^{(n-1)}(x) \neq 0 \neq G^{(n-1)}(x), x \in I$,

(iii) the quotients $f^{(n-1)} / g^{(n-1)}$ and $F^{(n-1)} / G^{(n-1)}$ have nonvanishing first derivative on $I$. Then, the equality

$$
\left[\begin{array}{l}
f \\
g
\end{array}\right]\left(x_{1}, x_{2}, \ldots, x_{n}\right)=\left[\begin{array}{l}
F \\
G
\end{array}\right]\left(x_{1}, x_{2}, \ldots, x_{n}\right), \quad x_{1}, x_{2}, \ldots, x_{n} \in I,
$$

holds if and only if there exist four constants $\alpha, \beta, \gamma, \delta$ with $\alpha \delta-\beta \gamma \neq 0$ such that for every $x \in I$,

$$
\begin{aligned}
& F^{(n-1)}(x)=\alpha f^{(n-1)}(x)+\beta g^{(n-1)}(x), \\
& G^{(n-1)}(x)=\gamma f^{(n-1)}(x)+\delta g^{(n-1)}(x) .
\end{aligned}
$$

In the previous work [10] by the same author, a representation result for two-variable weighted means of the type

$$
M_{\Phi, F}(x, y)=\Phi^{-1}\left(\frac{\Phi(x) F(x)+\Phi(y) F(y)}{F(x)+F(y)}\right)
$$

is obtained. There, after supposing a convenient regularity on the involved functions, the problem of identifying condition under which $M_{\Phi, F}(x, y) \equiv M_{\Psi, G}(x, y)$ is reduced to differential equations. In a subsequent paper (see [12]), Losonczi finally succeeded in practicing an analogous reduction for the problem of representation of two-variable Cauchy 
means. We will postpone our assessment of the Losonczi methods and results up to the last section of this paper. Besides what can be considered as a completion of Theorem 1.2 for the case $n=2$, a different strategy will be indicated in that section to tackle the problem of representation of two-variable Cauchy means as well as other similar representation problems.

\section{Proof of Theorem 1.1}

As a first step in proving Theorem 1.1, we are to derive from (1.2) and (1.3) a necessary differential condition of invariance. In this regard, observe that making $y \rightarrow x$ in (1.2) merely leads to the trivial identity

$$
\begin{aligned}
& \frac{X_{x}(f(x), g(x)) f^{\prime}(x)-X_{y}(f(x), g(x)) g^{\prime}(x)}{Y_{x}(f(x), g(x)) f^{\prime}(x)-Y_{y}(f(x), g(x)) g^{\prime}(x)} \\
& \quad=\frac{X_{x}(f(x), g(x)) f^{\prime}(x)-X_{y}(f(x), g(x)) g^{\prime}(x)}{Y_{x}(f(x), g(x)) f^{\prime}(x)-Y_{y}(f(x), g(x)) g^{\prime}(x)} .
\end{aligned}
$$

To overcome this difficulty, we recall the simple property of proportions which reads as

$$
\frac{a}{b}=\frac{c}{d} \Longrightarrow \frac{a-c}{b-d}=\frac{a}{b}
$$

so that from (1.2) and (1.3), we deduce

$$
\begin{aligned}
& \frac{X(f(y), g(y))-X(f(x), g(x))-X_{x}(f(\mu), g(\mu)) \Delta f-X_{y}(f(\mu), g(\mu)) \Delta g}{Y(f(y), g(y))-Y(f(x), g(x))-Y_{x}(f(\mu), g(\mu)) \Delta f-Y_{y}(f(\mu), g(\mu)) \Delta g} \\
& \quad=\frac{X(f(y), g(y))-X(f(x), g(x))}{Y(f(y), g(y))-Y(f(x), g(x))},
\end{aligned}
$$

where, for the sake of brevity, we have set

$$
\Delta f=f(y)-f(x), \quad \Delta g=g(y)-g(x) .
$$

Assuming that the transformation $\Phi=(X, Y)$ is $\mathscr{C}^{2}$, we pass to the limit $y \rightarrow x$ in (2.3) by repeatedly using l'Hospital rule and recalling the relationships

$$
\begin{gathered}
\mu_{x}(x, x)=\mu_{y}(x, x)=\frac{1}{2}, \\
\mu_{x x}(x, x)=\mu_{y y}(x, x)=-\mu_{x y}(x, x), \quad x \in I,
\end{gathered}
$$

which hold for every (sufficiently regular) symmetric mean $\mu$. In this way, we obtain

$$
\begin{aligned}
& \frac{\left[f^{\prime}(x)\right]^{2} X_{x x}(f(x), g(x))+2 f^{\prime}(x) g^{\prime}(x) X_{x y}(f(x), g(x))+\left[g^{\prime}(x)\right]^{2} X_{y y}(f(x), g(x))}{\left[f^{\prime}(x)\right]^{2} Y_{x x}(f(x), g(x))+2 f^{\prime}(x) g^{\prime}(x) Y_{x y}(f(x), g(x))+\left[g^{\prime}(x)\right]^{2} Y_{y y}(f(x), g(x))} \\
& \quad=\frac{f^{\prime}(x) X_{x}(f(x), g(x))+g^{\prime}(x) X_{y}(f(x), g(x))}{f^{\prime}(x) Y_{x}(f(x), g(x))+g^{\prime}(x) Y_{y}(f(x), g(x))} .
\end{aligned}
$$


Now, the arbitrariness of functions $f$ and $g$ (and therefore, that one corresponding to their derivatives) enables us to rewrite the equality (2.6) in the form

$$
\frac{A^{2} X_{x x}(x, y)+2 A B X_{x y}(x, y)+B^{2} X_{y y}(x, y)}{A^{2} Y_{x x}(x, y)+2 A B Y_{x y}(x, y)+B^{2} Y_{y y}(x, y)}=\frac{A X_{x}(x, y)+B X_{y}(x, y)}{A Y_{x}(x, y)+B Y_{y}(x, y)}, \quad(x, y) \in \mathbb{R}^{2},
$$

where $A, B \in \mathbb{R}$ do not vanish simultaneously. After simple algebraic manipulations, we see that this last condition is equivalent to the following one:

$$
U A^{3}+V A^{2} B+W A B^{2}+Z B^{3}=0, \quad A, B \in \mathbb{R} \backslash\{0\},
$$

where

$$
\begin{aligned}
U & =X_{x} Y_{x x}-Y_{x} X_{x x}, \\
V & =X_{y} Y_{y y}-Y_{y} X_{y y}, \\
W & =X_{y} Y_{x x}+2 X_{x} Y_{x y}-\left(Y_{y} X_{x x}+2 Y_{x} X_{x y}\right), \\
Z & =2 X_{y} Y_{x y}+X_{x} Y_{y y}-\left(2 Y_{y} X_{x y}+Y_{x} X_{y y}\right) .
\end{aligned}
$$

Hence, we derive the following system of PDEs:

$$
\begin{aligned}
X_{x} Y_{x x}-Y_{x} X_{x x} & =0 \\
X_{y} Y_{y y}-Y_{y} X_{y y} & =0 \\
X_{y} Y_{x x}+2 X_{x} Y_{x y} & =Y_{y} X_{x x}+2 Y_{x} X_{x y} \\
2 X_{y} Y_{x y}+X_{x} Y_{y y} & =2 Y_{y} X_{x y}+Y_{x} X_{y y}
\end{aligned}
$$

System (2.10) expresses the differential necessary conditions of invariance we sought for expression (1.1). Fortunately, all plane transformations with coordinate functions $X$, $Y$ satisfying this system can be elementarily computed. We are to perform this task in the next section, where we prove that the changes of coordinates solving system (2.10) coincide with the Lie group of transformations that leaves invariant the linear second-order ODE $y^{\prime \prime}=0$, a Lie group whose determining (linear) system exhibits some similitude with system (2.10) (cf. [6, page 122]).

\section{Solution of system (2.10)}

We are to determine the solutions $(X, Y)$ to system (2.10) which are changes of coordinates. To begin, from the first equation in (2.10), we obtain

$$
\frac{X_{x x}}{X_{x}}=\frac{Y_{x x}}{Y_{x}}
$$

and, after integration, we deduce

$$
Y_{x}(x, y)=\psi_{1}(y) X_{x}(x, y)
$$


2902 Invariance of the Cauchy mean-value expression

with $\psi_{1}$ an arbitrary function. Now, integrating (3.2), we deduce

$$
Y(x, y)=\psi_{1}(y) X(x, y)-\psi_{2}(y)
$$

where $\psi_{2}$ is a new arbitrary function. The second equation of system (2.10) can be integrated in a similar way:

$$
Y(x, y)=\phi_{1}(x) X(x, y)-\phi_{2}(x)
$$

with $\phi_{1}, \phi_{2}$ arbitrary functions. Thus, from (3.3) and (3.4), we derive

$$
X(x, y)=\frac{\psi_{2}(y)-\phi_{2}(x)}{\psi_{1}(y)-\phi_{1}(x)}
$$

In order to introduce (3.3), (3.4), and (3.5) into the third and fourth equations of system (2.10), we compute the first- and second-order partial derivatives of $X$ and $Y$ as follows:

$$
\begin{aligned}
Y_{x} & =\psi_{1}(y) X_{x}, \\
Y_{y} & =\phi_{1}(x) X_{y}, \\
Y_{x x} & =\psi_{1}(y) X_{x x}, \\
Y_{y y} & =\phi_{1}(x) X_{y y}, \\
Y_{x y} & =\psi_{1}^{\prime}(y) X_{x}+\psi_{1}(y) X_{x y}=\phi_{1}^{\prime}(x) X_{y}+\phi_{1}(x) X_{x y} .
\end{aligned}
$$

Once substituted these expressions in the third and fourth equations of (2.10), we obtain

$$
\begin{aligned}
& \left(\psi_{1}(y)-\phi_{1}(x)\right) X_{y} X_{x x}+2 \psi_{1}^{\prime}(y) X_{x}^{2}=0, \\
& \left(\psi_{1}(y)-\phi_{1}(x)\right) X_{x} X_{y y}-2 \phi_{1}^{\prime}(x) X_{y}^{2}=0 .
\end{aligned}
$$

Now, to compute the partial derivatives of $X$ as deduced from expression (3.5), it will be useful to define

$$
\begin{array}{r}
\Delta_{i}(x, y) \triangleq \psi_{i}(y)-\phi_{i}(x), \quad i=1,2, \\
u(x, y) \triangleq \phi_{2}^{\prime}(x) \Delta_{1}(x, y)-\phi_{1}^{\prime}(x) \Delta_{2}(x, y), \\
v(x, y) \triangleq \psi_{2}^{\prime}(y) \Delta_{1}(x, y)-\psi_{1}^{\prime}(y) \Delta_{2}(x, y) .
\end{array}
$$

Then, observing that

$$
\begin{aligned}
& \frac{\partial u}{\partial x}(x, y)=\phi_{2}^{\prime \prime}(x) \Delta_{1}(x, y)-\phi_{1}^{\prime \prime}(x) \Delta_{2}(x, y) \\
& \frac{\partial v}{\partial y}(x, y)=\psi_{2}^{\prime \prime}(y) \Delta_{1}(x, y)-\psi_{1}^{\prime \prime}(y) \Delta_{2}(x, y)
\end{aligned}
$$

and that

$$
\frac{\partial u}{\partial y}(x, y)=\phi_{2}^{\prime}(x) \psi_{1}^{\prime}(y)-\phi_{1}^{\prime}(x) \psi_{2}^{\prime}(y)=\frac{\partial v}{\partial x}(x, y)
$$


we obtain

$$
\begin{aligned}
& X_{x}=-\frac{u}{\Delta_{1}^{2}}, \\
& X_{y}=\frac{v}{\Delta_{1}^{2}}, \\
& X_{x x}=-\frac{\Delta_{1}(\partial u / \partial x)+2 \phi_{1}^{\prime}(x) u}{\Delta_{1}^{3}}, \\
& X_{y y}=\frac{\Delta_{1}(\partial v / \partial y)-2 \psi_{1}^{\prime}(y) v}{\Delta_{1}^{3}} .
\end{aligned}
$$

By substituting (3.12) and (3.13) in (3.7), we conclude that

$$
\begin{aligned}
& -\Delta_{1} v \frac{\partial u}{\partial x}+2 u\left(\psi_{1}^{\prime}(y) u-\phi_{1}^{\prime}(x) v\right)=0 \\
& -\Delta_{1} u \frac{\partial v}{\partial y}+2 v\left(\psi_{1}^{\prime}(y) u-\phi_{1}^{\prime}(x) v\right)=0,
\end{aligned}
$$

whence, taking into account that (3.9), (3.10), and (3.11) imply that

$$
\psi_{1}^{\prime}(y) u-\phi_{1}^{\prime}(x) v=\left(\phi_{2}^{\prime}(x) \psi_{1}^{\prime}(y)-\phi_{1}^{\prime}(x) \psi_{2}^{\prime}(y)\right) \Delta_{1}=\frac{\Delta_{1} \partial u}{\partial y}=\frac{\Delta_{1} \partial v}{\partial x},
$$

we derive

$$
\begin{aligned}
& \Delta_{1}\left(v \frac{\partial u}{\partial x}-2 u \frac{\partial v}{\partial x}\right)=0, \\
& \Delta_{1}\left(u \frac{\partial v}{\partial y}-2 v \frac{\partial u}{\partial y}\right)=0 .
\end{aligned}
$$

From (3.5), we see that $\Delta_{1}$ cannot vanish identically, and then (3.16) together with identity (3.11) provide

$$
\begin{gathered}
v \frac{\partial u}{\partial x}-2 u \frac{\partial v}{\partial x}=0 \\
u \frac{\partial v}{\partial y}-2 v \frac{\partial u}{\partial y}=0 \\
\frac{\partial u}{\partial y}=\frac{\partial v}{\partial x}
\end{gathered}
$$

Our next step will be to solve this system of first-order PDEs. For this purpose, let us rewrite the first equation in the form

$$
\frac{u_{x}}{u}=2 \frac{v_{x}}{v}
$$

which once integrated gives

$$
u(x, y)=q(y) v^{2}(x, y),
$$


2904 Invariance of the Cauchy mean-value expression

where $q$ is an arbitrary function. For the second equation in (3.17), we proceed in a similar way to obtain

$$
v(x, y)=p(x) u^{2}(x, y)
$$

with $p$ another arbitrary function. From (3.19) and (3.20), we deduce

$$
\begin{aligned}
& u\left(1-q(y) p^{2}(x) u\right)=0, \\
& v\left(1-q^{2}(y) p(x) v\right)=0 .
\end{aligned}
$$

In view of the fact that the pair of functions $(X, Y)$ is supposed to be a change of coordinates, its Jacobian $\partial(X, Y) / \partial(x, y)$ cannot vanish:

$$
\frac{\partial(X, Y)}{\partial(x, y)}=X_{x} Y_{y}-X_{y} Y_{x}=-\frac{u v}{\Delta_{1}^{3}} \neq 0,
$$

which shows that neither $u$ nor $v$ vanishes. Thus, from (3.21), we derive

$$
\begin{aligned}
& u(x, y)=\frac{1}{p^{2}(x) q(y)}, \\
& v(x, y)=\frac{1}{p(x) q^{2}(y)},
\end{aligned}
$$

and replacing the expressions (3.23) in the third equation from (3.17), we obtain

$$
-\frac{q^{\prime}(y)}{p^{2}(x) q^{2}(y)}=\frac{\partial u}{\partial y}=\frac{\partial v}{\partial x}=-\frac{p^{\prime}(x)}{p^{2}(x) q^{2}(y)}
$$

that is, for a real constant $\lambda$,

$$
p^{\prime}(x)=\lambda=q^{\prime}(y)
$$

or, integrating,

$$
p(x)=\lambda x+\alpha, \quad q(y)=\lambda y+\beta,
$$

where $\alpha, \beta \in \mathbb{R}$. A substitution of equalities (3.26) in (3.23) enables us to write the solutions $u, v$ to system (3.17) in the form

$$
\begin{aligned}
& u(x, y)=\frac{1}{(\lambda x+\alpha)^{2}(\lambda y+\beta)}, \\
& v(x, y)=\frac{1}{(\lambda x+\alpha)(\lambda y+\beta)^{2}} .
\end{aligned}
$$

Now, recalling the definition (3.9) of $u$ and $v,(3.27)$ can be rewritten as

$$
\begin{aligned}
& \phi_{2}^{\prime}(x) \Delta_{1}(x, y)-\phi_{1}^{\prime}(x) \Delta_{2}(x, y)=\frac{1}{(\lambda x+\alpha)^{2}(\lambda y+\beta)}, \\
& \psi_{2}^{\prime}(y) \Delta_{1}(x, y)-\psi_{1}^{\prime}(y) \Delta_{2}(x, y)=\frac{1}{(\lambda x+\alpha)(\lambda y+\beta)^{2}},
\end{aligned}
$$


a linear system of equations in $\Delta_{1}$ and $\Delta_{2}$ whose determinant is given by

$$
\begin{aligned}
\left|\begin{array}{ll}
\phi_{2}^{\prime}(x) & -\phi_{1}^{\prime}(x) \\
\psi_{2}^{\prime}(y) & -\psi_{1}^{\prime}(y)
\end{array}\right| & =\phi_{1}^{\prime}(x) \psi_{2}^{\prime}(y)-\phi_{2}^{\prime}(x) \psi_{1}^{\prime}(y) \\
& =-\frac{\partial u}{\partial y}=-\frac{\partial v}{\partial x}=\frac{\lambda}{(\lambda x+\alpha)^{2}(\lambda y+\beta)^{2}} .
\end{aligned}
$$

Two cases do appear in accordance with $\lambda \neq 0$ or $\lambda=0$; in what follows, we will consider them separately.

Case $1(\lambda \neq 0)$. In this case, system (3.28) is uniquely resolvable in the form

$$
\begin{aligned}
& \Delta_{1}(x, y)=\psi_{1}(y)-\phi_{1}(x)=\frac{1}{\lambda}\left((\lambda x+\alpha) \phi_{1}^{\prime}(x)-(\lambda y+\beta) \psi_{1}^{\prime}(y)\right), \\
& \Delta_{2}(x, y)=\psi_{2}(y)-\phi_{2}(x)=\frac{1}{\lambda}\left((\lambda x+\alpha) \phi_{2}^{\prime}(x)-(\lambda y+\beta) \psi_{2}^{\prime}(y)\right),
\end{aligned}
$$

or, setting $\alpha_{0}=\alpha / \lambda, \beta_{0}=\beta / \lambda$,

$$
\begin{aligned}
& \psi_{1}(y)+\left(y+\beta_{0}\right) \psi_{1}^{\prime}(y)=\phi_{1}(x)+\left(x+\alpha_{0}\right) \phi_{1}^{\prime}(x), \\
& \psi_{2}(y)+\left(y+\beta_{0}\right) \psi_{2}^{\prime}(y)=\phi_{2}(x)+\left(x+\alpha_{0}\right) \phi_{2}^{\prime}(x) .
\end{aligned}
$$

Reasoning as before with (3.25), we see that there exist two real constants $\rho_{1}$ and $\rho_{2}$ such that

$$
\begin{aligned}
& \left(y+\beta_{0}\right) \psi_{1}^{\prime}(y)+\psi_{1}(y)=\rho_{1}=\left(x+\alpha_{0}\right) \phi_{1}^{\prime}(x)+\phi_{1}(x), \\
& \left(y+\beta_{0}\right) \psi_{2}^{\prime}(y)+\psi_{2}(y)=\rho_{2}=\left(x+\alpha_{0}\right) \phi_{2}^{\prime}(x)+\phi_{2}(x) .
\end{aligned}
$$

The solution of these equations is a straightforward matter:

$$
\begin{array}{ll}
\psi_{1}(y)=\frac{\rho_{1} y+\gamma_{1}}{y+\beta_{0}}, & \phi_{1}(x)=\frac{\rho_{1} x+\gamma_{2}}{x+\alpha_{0}}, \\
\psi_{2}(y)=\frac{\rho_{2} y+\gamma_{3}}{y+\beta_{0}}, & \phi_{2}(x)=\frac{\rho_{2} x+\gamma_{4}}{x+\alpha_{0}},
\end{array}
$$

where the greek characters all denote real constants.

Finally, we replace (3.33) in (3.5) and (3.3) to obtain

$$
\begin{aligned}
& X(x, y)=\frac{\left(\gamma_{3}-\beta_{0} \rho_{2}\right) x+\left(\alpha_{0} \rho_{2}-\gamma_{4}\right) y+\left(\alpha_{0} \gamma_{3}-\beta_{0} \gamma_{4}\right)}{\left(\gamma_{1}-\beta_{0} \rho_{1}\right) x+\left(\alpha_{0} \rho_{1}-\gamma_{2}\right) y+\left(\alpha_{0} \gamma_{1}-\beta_{0} \gamma_{2}\right)} \\
& Y(x, y)=\frac{\left(\rho_{1} \gamma_{3}-\rho_{2} \gamma_{1}\right) x+\left(\rho_{2} \gamma_{2}-\rho_{1} \gamma_{4}\right) y+\left(\gamma_{2} \gamma_{3}-\gamma_{1} \gamma_{4}\right)}{\left(\gamma_{1}-\beta_{0} \rho_{1}\right) x+\left(\alpha_{0} \rho_{1}-\gamma_{2}\right) y+\left(\alpha_{0} \gamma_{1}-\beta_{0} \gamma_{2}\right)} .
\end{aligned}
$$

Case $2(\lambda=0)$. From (3.29), we obtain

$$
\phi_{1}^{\prime}(x) \psi_{2}^{\prime}(y)-\phi_{2}^{\prime}(x) \psi_{1}^{\prime}(y)=0
$$


2906 Invariance of the Cauchy mean-value expression

and therefore there exists $\rho \in \mathbb{R}$ such that

$$
\frac{\phi_{2}^{\prime}(x)}{\phi_{1}^{\prime}(x)}=\rho=\frac{\psi_{2}^{\prime}(y)}{\psi_{1}^{\prime}(y)}
$$

that is,

$$
\begin{aligned}
& \phi_{2}(x)=\rho \phi_{1}(x)+\alpha_{1}, \\
& \psi_{2}(y)=\rho \psi_{1}(y)+\beta_{1},
\end{aligned}
$$

for some real constants $\alpha_{1}, \beta_{1}$. Hence,

$$
\Delta_{2}(x, y)=\psi_{2}(y)-\phi_{2}(x)=\rho\left(\psi_{1}(y)-\phi_{1}(x)\right)+\left(\beta_{1}-\alpha_{1}\right)=\rho \Delta_{1}(x, y)+\left(\beta_{1}-\alpha_{1}\right),
$$

which shows that $\alpha_{1} \neq \beta_{1}$ (in other case, $X=\Delta_{2} / \Delta_{1} \equiv \rho$ and $(X, Y)$ would not be a change of coordinates). Replacing (3.36) and (3.38) in (3.28), we obtain

$$
\begin{aligned}
& \phi_{1}^{\prime}(x)\left(\alpha_{1}-\beta_{1}\right)=\frac{1}{\alpha^{2} \beta}, \\
& \psi_{1}^{\prime}(y)\left(\alpha_{1}-\beta_{1}\right)=\frac{1}{\alpha \beta^{2}},
\end{aligned}
$$

or, after integrating and renaming the constants

$$
\begin{aligned}
& \phi_{1}(x)=\alpha_{2} x+\beta_{2}, \\
& \psi_{1}(y)=\alpha_{3} y+\beta_{3} .
\end{aligned}
$$

Finally, from (3.5), (3.38), and (3.40), we deduce

$$
X(x, y)=\rho+\frac{\beta_{1}-\alpha_{1}}{\psi_{1}(y)-\phi_{1}(x)}=\frac{-\rho \alpha_{2} x+\rho \alpha_{3} y+\rho\left(\beta_{3}-\beta_{2}\right)+\left(\beta_{1}-\alpha_{1}\right)}{-\alpha_{2} x+\alpha_{3} y+\left(\beta_{3}-\beta_{2}\right)},
$$

while (3.4) yields

$$
\begin{aligned}
Y(x, y) & =\phi_{1}(y)\left(\rho+\frac{\beta_{1}-\alpha_{1}}{\psi_{1}(y)-\phi_{1}(x)}\right)-\phi_{2}(y)=-\alpha_{1}+\frac{\left(\beta_{1}-\alpha_{1}\right) \phi_{1}(x)}{\psi_{1}(y)-\phi_{1}(x)} \\
& =\frac{-\alpha_{1} \psi_{1}(y)+\beta_{1} \phi_{1}(x)}{\psi_{1}(y)-\phi_{1}(x)}=\frac{\beta_{1} \alpha_{2} x-\alpha_{1} \alpha_{3} y+\left(\beta_{1} \beta_{2}-\alpha_{1} \beta_{3}\right)}{-\alpha_{2} x+\alpha_{3} y+\left(\beta_{3}-\beta_{2}\right)} .
\end{aligned}
$$

Synthesizing the just-obtained results and studying the dependence of the real parameters which appear in (3.34) and (3.41)-(3.42), we see that every transformation $(X, Y)$ leaving expression (1.1) invariant must have the form

$$
\begin{aligned}
& X(x, y)=\frac{a_{1} x+b_{1} y+c_{1}}{a_{3} x+b_{3} y+c_{3}}, \\
& Y(x, y)=\frac{a_{2} x+b_{2} y+c_{2}}{a_{3} x+b_{3} y+c_{3}},
\end{aligned}
$$


where

$$
\left|\begin{array}{lll}
a_{1} & b_{1} & c_{1} \\
a_{2} & b_{2} & c_{2} \\
a_{3} & b_{3} & c_{3}
\end{array}\right| \neq 0
$$

and only eight from the nine real parameters are independent of each other. In (3.43), the 8-parameter plane projective group is recognized, but simple examples show that $a$ projective transformation does not generally leave expression (1.1) invariant. In the next section, the precise restrictions to be imposed on a projective transformation in order that it leaves expression (1.1) invariant will be discussed and the proof of Theorem 1.1 will be finished.

\section{Completion of the proof}

Now, we complete the proof of Theorem 1.1. That every affine transformation leaves expression (1.1) invariant was just proved in Section 1, so that it remains only to establish the converse. The previous developments show that a $\mathscr{C}^{2}$ transformation $\Phi=(X, Y)$ leaving expression (1.1) invariant must be a projective one; then, replacing $X$ and $Y$ given by (3.43) in (1.2) and (1.3) and after making some algebraic manipulations, we see that a projective transformation leaves expression (1.1) invariant provided that for very pair $f$, $g$ differentiable function with $f^{\prime} / g^{\prime}$ being strictly monotone, the equality

$$
\frac{\alpha_{1} \Delta g-\beta_{1} \Delta f+\gamma_{1}(g(x) \Delta f-f(x) \Delta g)}{\alpha_{2} \Delta g-\beta_{2} \Delta f+\gamma_{2}(g(x) \Delta f-f(x) \Delta g)}=\frac{\alpha_{1} \Delta g-\beta_{1} \Delta f+\gamma_{1}(g(\mu) \Delta f-f(\mu) \Delta g)}{\alpha_{2} \Delta g-\beta_{2} \Delta f+\gamma_{2}(g(\mu) \Delta f-f(\mu) \Delta g)}
$$

holds. In (4.1), $\mu=\mu(x, y)$ is defined by (1.3), $\Delta f$ and $\Delta g$ are given by (2.4) and, moreover, we have set

$$
\alpha_{i}=\left|\begin{array}{ll}
b_{i} & c_{i} \\
b_{3} & c_{3}
\end{array}\right|, \quad \beta_{i}=-\left|\begin{array}{ll}
a_{i} & c_{i} \\
a_{3} & c_{3}
\end{array}\right|, \quad \gamma_{i}=\left|\begin{array}{ll}
a_{i} & b_{i} \\
a_{3} & b_{3}
\end{array}\right|, \quad i=1,2
$$

From the arbitrariness of $f$ and $g$, we conclude that equality (4.1) holds if, and only if,

$$
\begin{aligned}
& \alpha_{1} \gamma_{2}=\alpha_{2} \gamma_{1}, \\
& \beta_{1} \gamma_{2}=\beta_{2} \gamma_{1} .
\end{aligned}
$$

By using some vector algebra, we can rewrite conditions (4.3) in terms of the coefficients of the projective transformation. In fact, defining $v_{i} \triangleq\left(a_{i}, b_{i}, c_{i}\right), i=1,2,3$, we have

$$
\left(\alpha_{i}, \beta_{i}, \gamma_{i}\right)=v_{i} \wedge v_{3}, \quad i=1,2,
$$

(where, as usual, $\wedge$ denotes the wedge product), and therefore

$$
\begin{aligned}
\left(v_{1} \wedge v_{3}\right) \wedge\left(v_{2} \wedge v_{3}\right) & =\left(\alpha_{1}, \beta_{1}, \gamma_{1}\right) \wedge\left(\alpha_{2}, \beta_{2}, \gamma_{2}\right) \\
& =\left(\beta_{1} \gamma_{2}-\beta_{2} \gamma_{1}, \alpha_{2} \gamma_{1}-\alpha_{1} \gamma_{2}, \alpha_{1} \beta_{2}-\alpha_{2} \beta_{1}\right)
\end{aligned}
$$


2908 Invariance of the Cauchy mean-value expression

But, from the identity

$$
\left(w_{1} \wedge w_{2}\right) \wedge\left(w_{3} \wedge w_{4}\right)=\operatorname{det}\left[w_{1}, w_{2}, w_{4}\right] w_{3}-\operatorname{det}\left[w_{1}, w_{2}, w_{3}\right] w_{4}
$$

which is valid for any four vectors $w_{i}$, we obtain

$$
\left(v_{1} \wedge v_{3}\right) \wedge\left(v_{2} \wedge v_{3}\right)=\operatorname{det}\left[v_{1}, v_{2}, v_{3}\right] v_{3}
$$

Since $\operatorname{det}\left[v_{1}, v_{2}, v_{3}\right] \neq 0$ by (3.44), from (4.5) and (4.7), we conclude that (4.3) is equivalent to

$$
a_{3}=0=b_{3}
$$

The proof of Theorem 1.1 finishes by merely observing that a projective transformation satisfying conditions (4.8) is an affine transformation.

\section{Applications and final discussions}

In this section, we will focalize on the problem of representation of Cauchy means. In the first place, we restate Theorem 1.1 in a way better adapted to our notational frame. To this end, we recall from Section 1 that for a pair $f, g: I \rightarrow \mathbb{R}$ of strictly monotonic and continuous functions, the Cauchy mean $\left[\begin{array}{l}f \\ g\end{array}\right]$ gives the Cauchy "intermediate value" corresponding to the plane curve $\left(\int_{a}^{t} f(\xi) d g(\xi), g(t)\right), t \in I$.

Theorem 5.1. Let $\Phi=(X, Y)$ be a $\mathscr{C}^{2}$ change of coordinates in the plane. Then, the equality of Cauchy means

$$
\left[\begin{array}{l}
X\left(\int_{a}^{t} f(\xi) d g(\xi), g(t)\right) \\
Y\left(\int_{a}^{t} f(\xi) d g(\xi), g(t)\right)
\end{array}\right]=\left[\begin{array}{l}
f \\
g
\end{array}\right]
$$

holds for every pair $f, g: I \rightarrow \mathbb{R}$ of strictly monotonic and continuous functions if and only if $\Phi$ is an affine change of coordinates given by (1.4).

A proof for this result easily follows from Theorem 1.1. In practice, Theorem 5.1 provides, for a given Cauchy mean $\left[\begin{array}{l}f \\ g\end{array}\right]$, a four-parameter family of Cauchy means $\left[\begin{array}{c}F \\ G\end{array}\right]$ such that $\left[\begin{array}{c}F \\ G\end{array}\right]=\left[\begin{array}{c}f \\ g\end{array}\right]$. In fact, we have

$$
\begin{gathered}
\int^{x} F d G=a_{1} \int^{x} f d g+b_{1} g(x)+c_{1}, \\
G(x)=a_{2} \int^{x} f d g+b_{2} g(x)+c_{2},
\end{gathered}
$$

or, by taking differentials,

$$
\begin{gathered}
F d G=a_{1} f d g+b_{1} d g, \\
d G=a_{2} f d g+b_{2} d g(x),
\end{gathered}
$$


whence we obtain

$$
F=\frac{a_{1} f+b_{1}}{a_{2} f+b_{2}} .
$$

Equalities (5.5) and (5.3) give us

$$
\begin{gathered}
F=\frac{a_{1} f+b_{1}}{a_{2} f+b_{2}}, \\
G=a_{2} \int^{x} f d g+b_{2} g(x)+c_{2},
\end{gathered}
$$

where only four parameters are independent.

Theorem 1.1 (or Theorem 5.1) can be considered as a suitable completion of Theorem 1.2 for the case of two-variable Cauchy means. An additional negative restatement of our result may be useful to clarify this point. Indeed, we can say that changes of coordinates plainly fail in furnishing all (two-variable) Cauchy means equal to a given Cauchy mean; hence, a sense can be conveyed to the case $n=2$ of Theorem 1.2 by observing that the statement is true also for $n=2$ provided that the pair of functions $(F, G)$ is the image of the pair $(f, g)$ under a $C^{2}$ change of coordinates in $\mathbb{R}^{2}$.

Now we turn slightly aside to discuss the solution to the representation problem for the two-variable Cauchy means as presented by Losonczi in [12]. By means of a clever procedure, in this paper, the author reduces the problem to solve the Riccati equation

$$
4 h^{\prime}=2 h^{2}+C\left(G^{\prime}\right)^{4 / 3},
$$

where $C$ is an arbitrary constant and $G$ is a solution of the fourth-order nonlinear equation

$$
9 \frac{G^{I V}}{G^{\prime}}-45 \frac{G^{\prime \prime} G^{\prime \prime \prime}}{\left(G^{\prime}\right)^{2}}+40\left(\frac{G^{\prime \prime}}{G^{\prime}}\right)^{3}=0 .
$$

In this way, 32 new families of solutions (apart from the "main family" expressed by (5.6)) arise for the problem of representation of two-variable Cauchy means.

When applied to an abstract functional equation, the method of reduction to differential equations often leads to a reasonable solution in the class of sufficiently regular functions (cf. the general discussion on the method in [1, pages 188-190]). However, if the functional equation to be solved possesses a strong geometric flavor (and (1.17) is a good example of this), a mere exhibition of the family of its solutions derived by reducing it and then solving a differential equation may supply an insufficient insight. We can demand, for instance, why the (regular) solutions to (1.17) can be grouped in 33 functionally different families and why, so to speak, not in 45. Furthermore, after the considerable technical efforts unfolded in [12] to furnish a "complete" solution for the problem of representation of two-variable Cauchy means, no particular knowledge is attained so as to distinguish its solutions from that ones obtained (by the same method) in [10] for 
the problem of representation of the weighted means (1.21). Now, Theorem 1.1 can be seen as a first step in gaining the just-mentioned insight through the use of theoretical tools which are more adequate to attack our problem than reduction to ODEs. After Theorem 1.1, we know that when acting on a given Cauchy mean, no group of "point transformations" of the plane (see [6] for the exact sense of this terminology) is capable to give all Cauchy means equal to a given Cauchy mean. Thus, in a second step, we are naturally compelled to define and then compute more general transformations leaving expression (1.1) invariant. Namely, we are thinking in contact transformations of the type

$$
\begin{aligned}
& F=X\left(f, g, f^{\prime}, g^{\prime}\right), \\
& G=Y\left(f, g, f^{\prime}, g^{\prime}\right) .
\end{aligned}
$$

It can be reasonably assumed that such a transformation satisfies the contact condition (cf. [6]). In principle, invariance under these transformations may explain the appearance of exceptional families of solutions to the problem of representation of two-variable means (Cauchy means or the so-called weighted means, among many others). In attempting a comparison amongst our method and reduction to ODEs, we find scarcely advisable the final need to solve a system of PDEs instead of an ODE. However, in a like manner as was found in the simple case studied in this paper, the equations in the resulting system are expected to possess a high degree of symmetry, and therefore the prospective of finding their solutions in closed form is vacuous at all. A minute development of these ideas will be the subject matter of a forthcoming paper.

The method of reduction to ODEs employed by Losonczi and the method which is based on the study of invariance of expression (1.1) under general transformations which is presented in this paper, both are in need of heavy regularity hypotheses on the involved functions. In this respect, a brief exposition of Matkowski's direct approach may be useful and clarifying. As claimed in [14] (and showed a few lines below), the problem of representation of two-variable Cauchy means can be reduced to solve the functional equation

$$
\phi\left(\frac{f(y)-f(x)}{y-x}\right)=\frac{g(y)-g(x)}{h(y)-h(x)} .
$$

For the case of Lagrangian means, this functional equation becomes

$$
\phi\left(\frac{f(y)-f(x)}{y-x}\right)=\frac{g(y)-g(x)}{y-x},
$$

an equation which, under very general hypotheses, can be locally reconducted to a Jensen equation for the function $\phi$ (cf. [13]). This procedure provides an alternative way to prove, for Lagrangian means, the representation result expressed by (1.11). Now, in extending this method to (5.10) (see [14]), some restrictive convexity hypotheses are needed whose fulfilment narrows the generality of the obtained results, which do not suffice to treat the problem of representation in full generality. In fact, a capital result in [14] reads, omitting unnecessary details, as follows. 
Theorem 5.2. Let $I \subseteq \mathbb{R}$ be an open interval. Suppose that the functions $f, g, h: I \rightarrow \mathbb{R}$ are strictly monotonic and differentiable, $f^{\prime}$ and $g^{\prime} / h^{\prime}$ are strictly monotonic, and $h^{\prime}, g^{\prime}, g^{\prime} / f^{\prime}$ are monotonic. Then $f, g, h$, and $\phi$ satisfy (5.10) if, and only if, there are $a, b, c, d, m, p, q, r \in$ $\mathbb{R} ; a d-b c \neq 0 ; m \neq 0$, such that

$$
\begin{gathered}
\phi(u)=\frac{a u+b}{c u+d}, \\
g(x)=m(a f(x)+b x)+p, \quad h(x)=m(c f(x)+d x)+q, \quad x \in I .
\end{gathered}
$$

Using (1.7), it is not difficult to see that even simple instances of equality of Cauchy means turn out to be out of reach of results like the previous one. For the geometric mean, we have (by abusing the notation once more time)

$$
\left[\begin{array}{l}
\ln x \\
\ln x
\end{array}\right](x, y)=\sqrt{x y}=\left[\begin{array}{c}
1 / x^{2} \\
x
\end{array}\right](x, y), \quad x, y>0,
$$

and the results in [14] do not apply to the functions involved (through (1.7)) in this identity. In the example, we have for $x>0$,

$$
f(x)=-\frac{1}{x}, \quad g(x)=\frac{1}{2} \ln ^{2} x, \quad h(x)=\ln x,
$$

while

$$
\phi(u)=-\frac{1}{2} \ln u
$$

Summarizing the above discussion, we can say that, up to date, a complete list of solutions is known at the best for the problem of representation of two-variable Cauchy means (as well as other lists for similar problems) but, on one hand, strong and extraneous regularity assumptions on the involved functions are needed to obtain such solutions and, on the other hand, the exact knowledge of the list provides a scarce enlightenment on the profound nature of the solutions.

A few final words follow on the problem of parameterization. By identity (1.12), a change of parameter $t=\phi(\tau)$ in the curve $(f(t), g(t))$ has the effect of conjugating the Cauchy mean $\mu=\left[\begin{array}{l}f \\ g\end{array}\right]$. Hence, a change of parameter $\phi$ will leave the mean $\mu$ invariant if, and only if, function $\phi$ is a solution of the functional equation

$$
\mu(\phi(x), \phi(y))=\phi(\mu(x, y))
$$

in the class of the homeomorphisms of the interval $I$. Equation (5.16) can be seen as a generalization of the Jensen equation and it has deserved a considerable study. In this respect, we refer the interested reader to the references $[1,2]$.

\section{Acknowledgments}

The subject matter of this paper was evolving from my correspondence with Julio Moro along the years 1999 and 2000. I am very grateful to L. Losonczi and J. Matkowski, who kindly provided me with copies of their works. 


\section{Invariance of the Cauchy mean-value expression}

\section{References}

[1] J. Aczél, Lectures on Functional Equations and Their Applications, Mathematics in Science and Engineering, vol. 19, Academic Press, New York, 1966.

[2] L. R. Berrone and A. L. Lombardi, A note on equivalence of means, Publ. Math. Debrecen 58 (2001), no. 1-2, 49-56.

[3] L. R. Berrone and J. Moro, Lagrangian means, Aequationes Math. 55 (1998), no. 3, 217-226.

[4] - On means generated through the Cauchy mean value theorem, Aequationes Math. 60 (2000), no. 1-2, 1-14.

[5] J. Blasińska and J. Matkowski, When Lagrangean and quasi-arithmetic means coincide, to appear.

[6] G. W. Bluman and S. Kumei, Symmetries and Differential Equations, Applied Mathematical Sciences, vol. 81, Springer, New York, 1989.

[7] P. S. Bullen, D. S. Mitrinović, and P. M. Vasić, Means and Their Inequalities, Mathematics and Its Applications (East European Series), vol. 31, D. Reidel, Dordrecht, 1988.

[8] C. Jordan, Calculus of Finite Differences, 2nd ed., Chelsea, New York, 1950.

[9] E. B. Leach and M. C. Sholander, Multivariable extended mean values, J. Math. Anal. Appl. 104 (1984), no. 2, 390-407.

[10] L. Losonczi, Equality of two variable weighted means: reduction to differential equations, Aequationes Math. 58 (1999), no. 3, 223-241.

[11] Equality of Cauchy mean values, Publ. Math. Debrecen 57 (2000), no. 1-2, 217-230.

[12] Equality of two variable Cauchy mean values, Aequationes Math. 65 (2003), no. 1-2, 61-81.

[13] J. Matkowski, Mean value property and associated functional equations, Aequationes Math. 58 (1999), no. 1-2, 46-59.

[14]_, Functional equation involving difference quotients - an envelope method, preprint.

Lucio R. Berrone: Consejo Nacional de Investigaciones Científicas y Técnicas, Departamento de Matemática, Facultad de Ciencias Exactas, Ingenieria y Agrimensura, Universidad Nacional de Rosario, Avenue Pellegrini 250, 2000 Rosario, Argentina

E-mail address: berrone@fceia.unr.edu.ar 


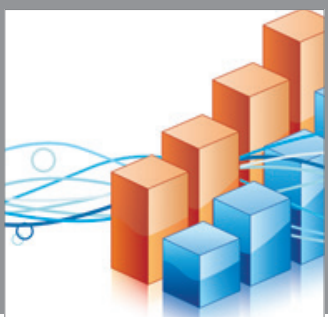

Advances in

Operations Research

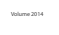

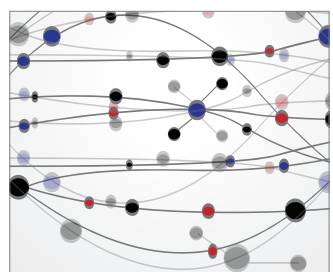

\section{The Scientific} World Journal
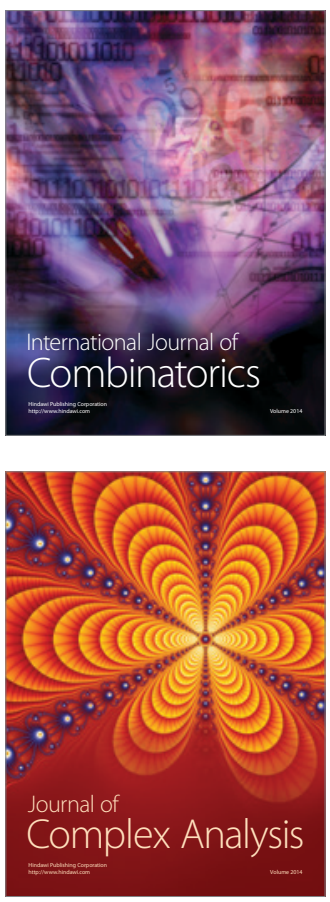

International Journal of

Mathematics and

Mathematical

Sciences
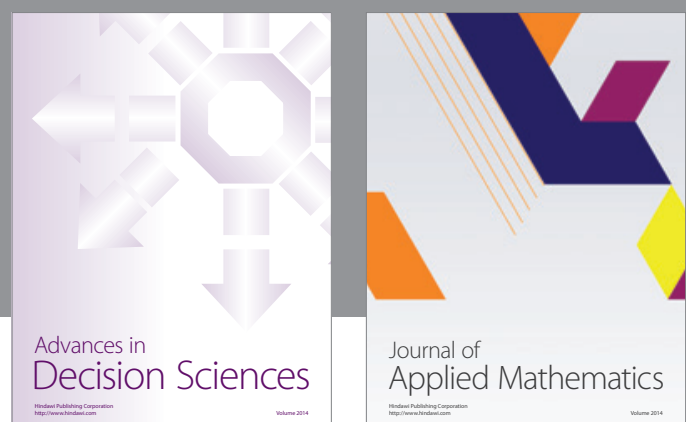

Journal of

Applied Mathematics
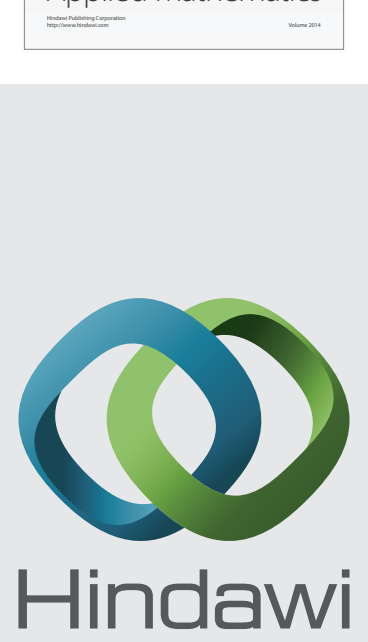

Submit your manuscripts at http://www.hindawi.com
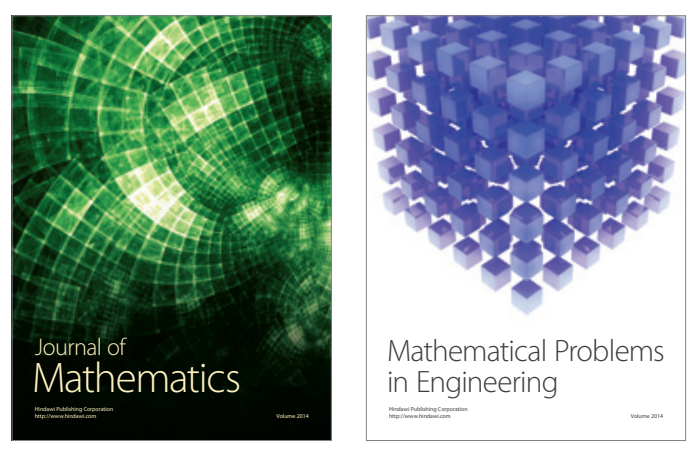

Mathematical Problems in Engineering
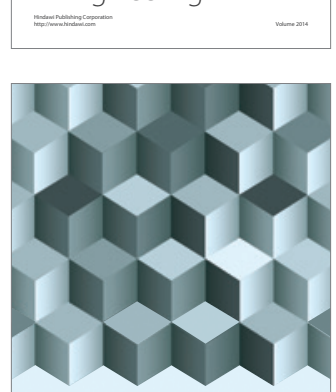

Journal of

Function Spaces
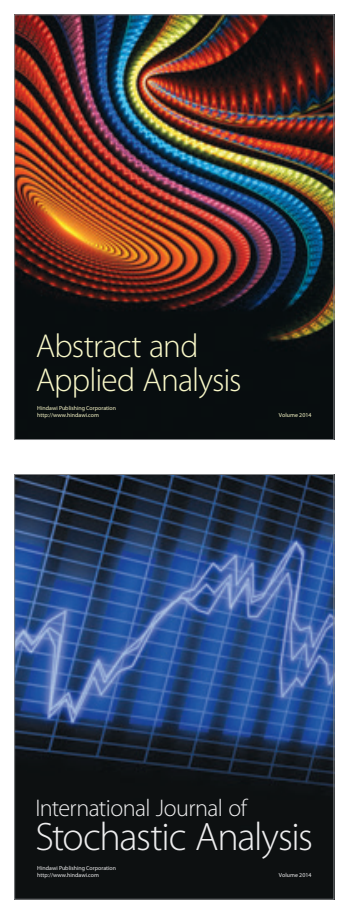

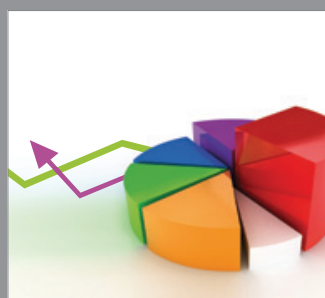

ournal of

Probability and Statistics

Promensencen
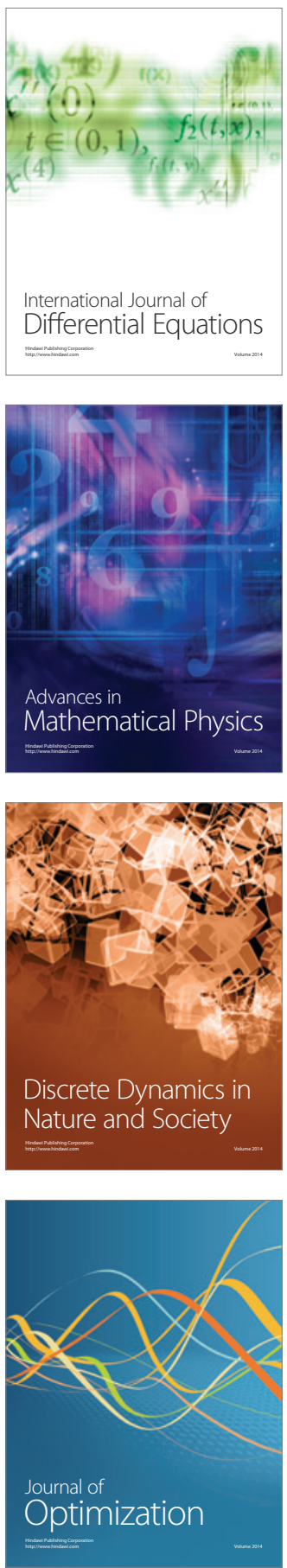